\title{
Automated Access Planning On Construction Sites: An Expert GIS Approach
}

\author{
Koshy Varghese a and James T. O'Connor ${ }^{\mathrm{b}}$ \\ a Department of Civil Engineering, The University of Texas at Austin, Austin, Texas 78712. \\ b Department of Civil Engineering, The University of Texas at Austin, Austin, Texas 78712.
}

\begin{abstract}
The use of prefabrication to enhance constructability necessitates careful planning for access of the prefabricated modules within a construction site. Current planning tools are inadequate for representing the integrated information required for planning access. Hence, developing a reliable plan using these tools is time consuming. This paper presents a computerized approach for planning vehicle access routes on an industrial site. It proposes that a Geographic Information System (GIS) be used to represent integrated information and an Expert System be used to represent the knowledge required to select the optimal access route for prefabricated modules on a construction site. The paper also presents the design of such a system using the GIS Arc/Info and the Expert System Nexpert Object.
\end{abstract}

\section{INTRODUCTION}

Prefabrication is an important constructability concept which when properly planned can enhance chances of project success [1]. The primary advantages of prefabrication are that it permits the manufacture of important components in a controlled environment and it permits the acceleration of construction schedule. But, to derive the benefits of prefabrication careful planning is necessary to ensure that the module can be transported to the site, transported to its final location within the site and installed without any hindrances. This study focuses on developing a computerized method for planning the transport of large prefabricated or preassembled modules within the site.

Large prefabricated or preassembled modules form an integral part of the process equipment at industrial facilities. These modules are usually very bulky and can weigh up to 10()()T. Figure 1. shows a prefabricated process column which was installed at a plant in Texas. This column weighs 6()()T and has dimensions of 210 ' $\times 3()^{\prime} \times 28$ '.

Planning the access of such modules within an industrial site is a challenging task for both new and retrofit projects [2] [3]. For new projects, the planners and designers have to synchronize decisions on site layout, facility design, construction sequence and vehicle characteristics to ensure that an accessible route to transport the module to its destination is available when the module is delivered to the site. For retrofit projects, the layout and the design of the existing facility is fixed, but the size of the modules can be varied. As the 
facility already exists and can be in operation, the planner has to determine the most suitable access route available for the transport of the module.

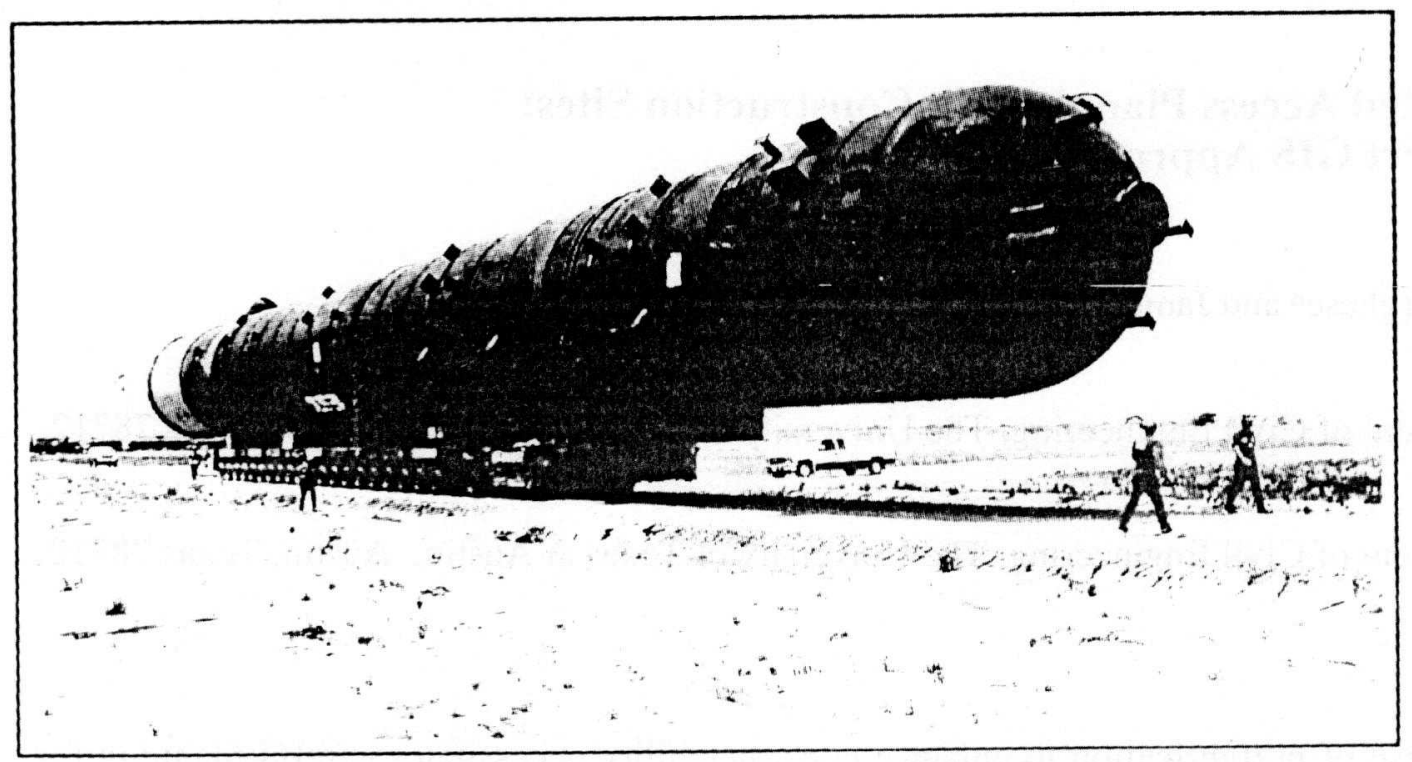

Figure 1. Transport of a prefabricated process column [Mammoet Transport]

To develop the best overall access plan, it is essential to investigate a number of alternate project scenarios. But the repetitive, tedious and time consuming nature of the analysis using current tools restricts the planners from investigating alternate scenarios. This can lead to the development of non-optimal or even erroneous plans.

The objective of this work is to investigate the feasibility of automating the repetitive planning tasks, thus enabling the planner to focus on the more creative components of the planning process.

The subsequent sections of the paper are presented in five parts. The next section discusses the variables and decisions influencing the planning process. The third section presents the basic concepts of Expert GIS and describes the tools used to implement it. The fourth section presents a conceptual design of the route planning system and the final section presents the conclusions of this paper.

\section{ACCESS PLANNING ISSUES}

The objective of planning access routes is to determine the optimal route for a vehicle traveling between two points on a construction site. The layout of the site for a new project will be dynamic as it will change as the construction progresses. The optimal route in such a case, can be defined as the route which satisfies both the access objective and overall project objectives. The access objective is to ensure that the transport activity has a minimum potential of heing obstructed from the various site features. The overall project objectives are to minimize cost and duration while ensuring a safe work environment.

The project management decisions influencing access are: site layout, facility design, construction sequence and transport vehicle characteristics. These decisions can be varied and combined to generate alternate project scenarios. Each scenario is then be analyzed for 
access requirements and the scenario which best satisfies the overall project objectives as well as the access objectives is selected for execution.

The human planner develops alternate scenarios based on his/her experience, imagination and creative thinking. To analyze a scenario the planner has to be aware of the project features which influence access and the methods to determine the potential of obstruction from these features. The common causes of obstruction on an industrial site are shown in Figure 2.

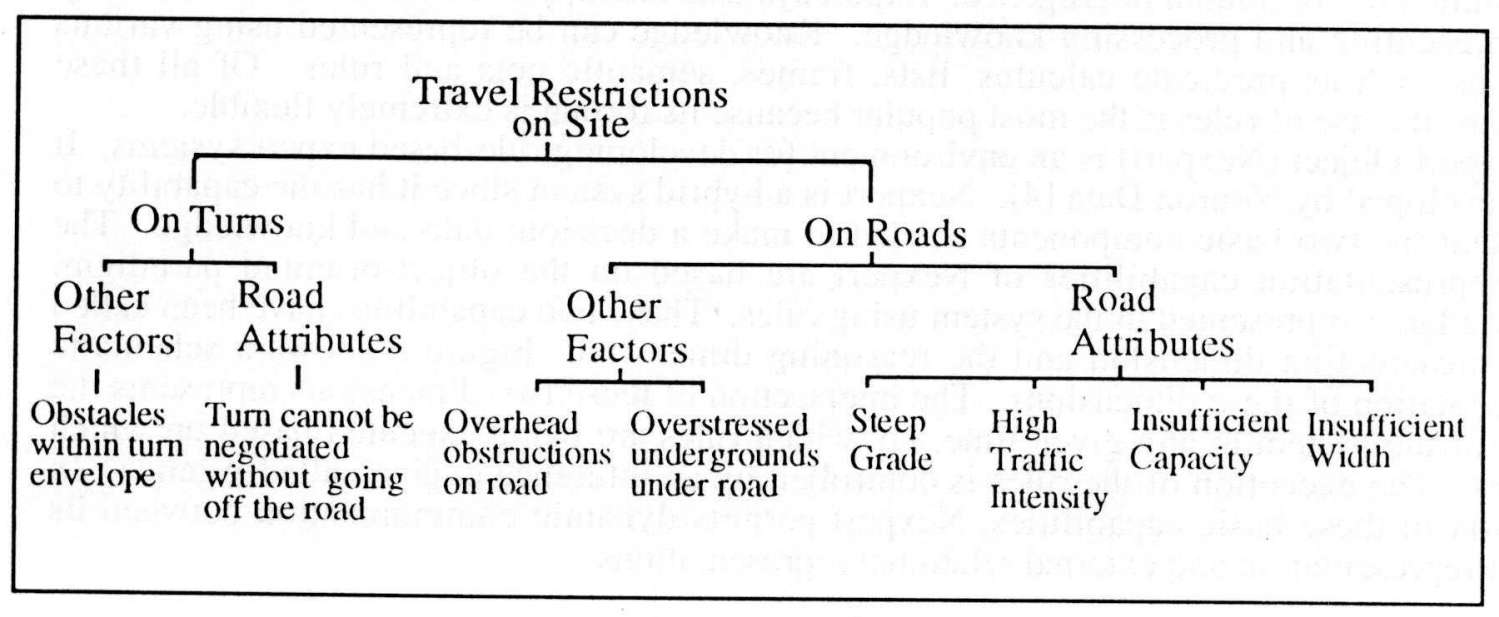

Figure 2. Causes of obstruction on an industrial site

The conventional tools used for analysis are: plot plans or solid models for representing layout of the site, design in the form of drawings, bar charts for representing the schedule, and templates to represent the vehicle/module. To analyze a given scenario, the planner has to refer to the schedule documents and design drawings to determine the layout of the site at the time of delivery and transform this information into a graphic representation. Next, the planner identifies potential access routes and using the template traces the vehicle paths along the routes to determine if they are obstructed. In addition calculations have to be performed to determine obstructions from features such as underground elements. If no accessible routes are found, elements of the scenario have to be modified to create new scenario which is then reanalyzed. If multiple access routes are found the planner has to compare the routes to select the best route. The comparison is based on the potential of obstruction from the features along the route and the route with the least potential of being obstructed is selected as the best route. Thus it can be seen that as the information is not integrated, keeping track of the various pieces of data is a tedious task. Further, the iterative nature of the process requires the planner to repeat many of the mundane tasks, thus reducing the planning productivity.

The successful development of such a system which automates the tedious and repetitive planning tasks will increase the planners productivity. Although the generation of a scenario is a challenging task it is based primarily on the human creative capabilities and no computer methods to date can accurately model these capabilities. Further, there are numerous other factors which influence the acceptability of a scenario. Thus, this work focuses on providing the planner tools using which he/she can use to: easily model alternate scenarios, represent the integrated information for each scenario and automatically analyze the scenarios for the selection of the best access routes. 


\section{EXPERT GIS CONCEPTS AND TOOLS}

An expert geographic information system combines the reasoning capability of an expert system and the spatial data representation and analysis capability of a geographic information system to form a powerful tool for spatial reasoning. The concept of expert GIS has been an active area of research, but only recent advances in hardware and software has permitted its application of to real world problems.

Expert systems is a branch of artificial intelligence, an area in computer science dealing with simulation of human intelligence. Expert systems attempt to simulate human reasoning by representing and processing knowledge. Knowledge can be represented using various methods such as predicate calculus, lists, frames, semantic nets and rules. Of all these methods, the use of rules is the most popular because its format is extremely flexible.

Nexpert Object (Nexpert) is an environment for developing rule-based expert systems. It was developed by Neuron Data [4]. Nexpert is a hybrid system since it has the capability to represent the two basic components needed to make a decision: data and knowledge. The data representation capabilities of Nexpert are based on the object-oriented paradigm. Knowledge is represented in the system using rules. These two capabilities have been called the representation dimension and the reasoning dimension. Figure 3 shows a schematic representation of these dimensions. The intersection of these two dimensions represents the focus of the system at any given time, i.e. which rules are being executed based on which objects. The execution of the rules is controlled by an inference engine called Agenda. In addition to these basic capabilities, Nexpert permits dynamic communication between its object representation and external relational representations.

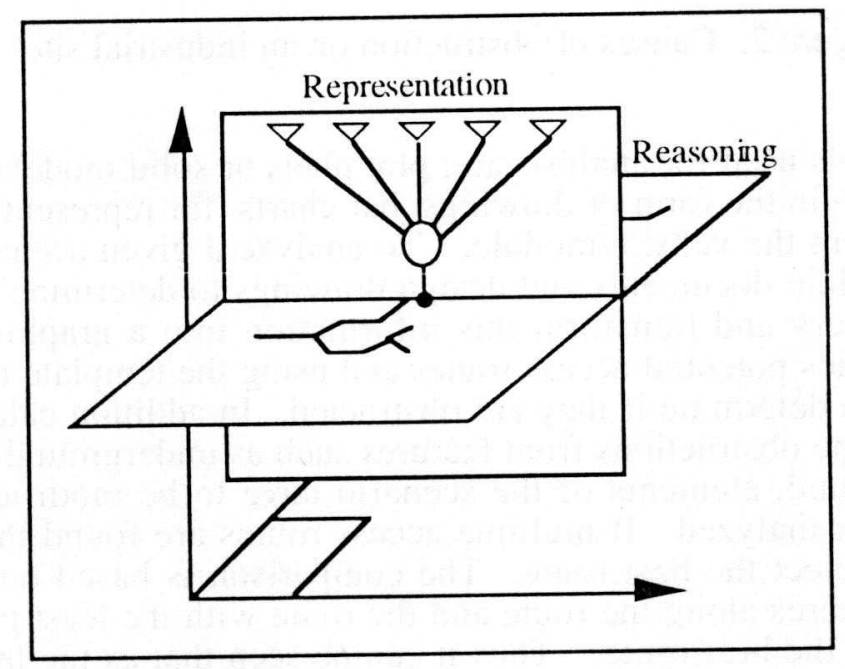

Figure 3. Nexpert Dimensions [4]

A geographic system is defined by Morehouse as a spatial database with a set of spatial operators [5]. In its basic form, it integrates spatial modeling, database management, spatial analysis and computer graphics into a software environment for representing and managing geographic features. Geographic information systems as a technology, had its origins in automated mapping systems about two decades ago.

The primary function of a geographic information system is to integrate and store locational (geometric) information and thematic (attribute) information. Locational 
information consists of data on the geographic location of points and lines, while thematic information consists of the non geometric attributes of the points and lines. For example, the locational information of a building can be the end coordinates of the lines which form its border, while the thematic information can pertain to its street address. Various sophisticated tools have been built to manipulate, query and analyze the integrated information. These tools along with the tools required to represent the information constitute a geographic information system.

Arc/Info is a commercial vector based geographic information system developed by Environmental Systems Research Institute [6]. Arc/Info's name is derived from the capability of the software to represent locational data (Arc), and thematic data (Info). In Arc/Info locational data is represented using a topological data model while the thematic data is represented using a relational model. This dual data model is called a geo-relational model, as it combines a specialized geographic view of the data with a conventional relational model. The numerous functions of Arc/Info are organized into various components, Table 1 presents the components of Arc/Info and their functions.

Table 1.

Arc/Info component and functions

\begin{tabular}{ll}
\hline Component & Function \\
\hline Arccad & Geometry creation and editing using CAD \\
Arcedit & Geomerty creation and editing using digitizing \\
Data Convertion & Coversion of foreign geometry formats \\
Stärter Kit & Bassic capabilities for representing information \\
Tables & Editing and adding non-geometric information \\
Arcplot & Graphical querying of represented information \\
Overlay & Spatial analysis of represented geometries \\
Network & Representation and analysis of networks \\
Macro Language & Creation of macro functions \\
\hline
\end{tabular}

There are two methods by which Nexpert and Arc/Info can be combined to form an expert geographic information system. The first method involves establishing a data link between the two software packages. Each software would share common data files but function independent of each other. As Arc/Info represents the attributes of a feature in a Dbase file format and Nexpert's databridge is capable of reading/writing information from/to this format, this link can be established by programming the rulebase to read the data, reason based on the data and write the conclusions back into the Arc/Info files.

The second method utilizes the "open" nature of both the systems to build an active command interface. This enables one software package to control the functioning of the other as well as the transfer of data between the two. Such an interface would be capable of dual control: Arc/Info can control Nexpert if the problem consists of a simple reasoning process based on intensive spatial analysis and Nexpert can control Arc/Info if the problem consists of a complex reasoning process using little spatial data [7]. 
The human planner, when planning for access, uses many capabilities which can be emulated by an expert GIS. Therefore, a computerized system developed using an expert GIS approach can assist the planner by automating many of the route planning tasks.

\section{SYSTEM DESIGN}

The overall design of the system is based on representing the roads on the site as a network, assigning travel impedances to the roads and turns and using a network search algorithm to determine the path of least impedance between any two points on the network. The overall flow chart required to utilize this approach is illustrated in Figure 4.

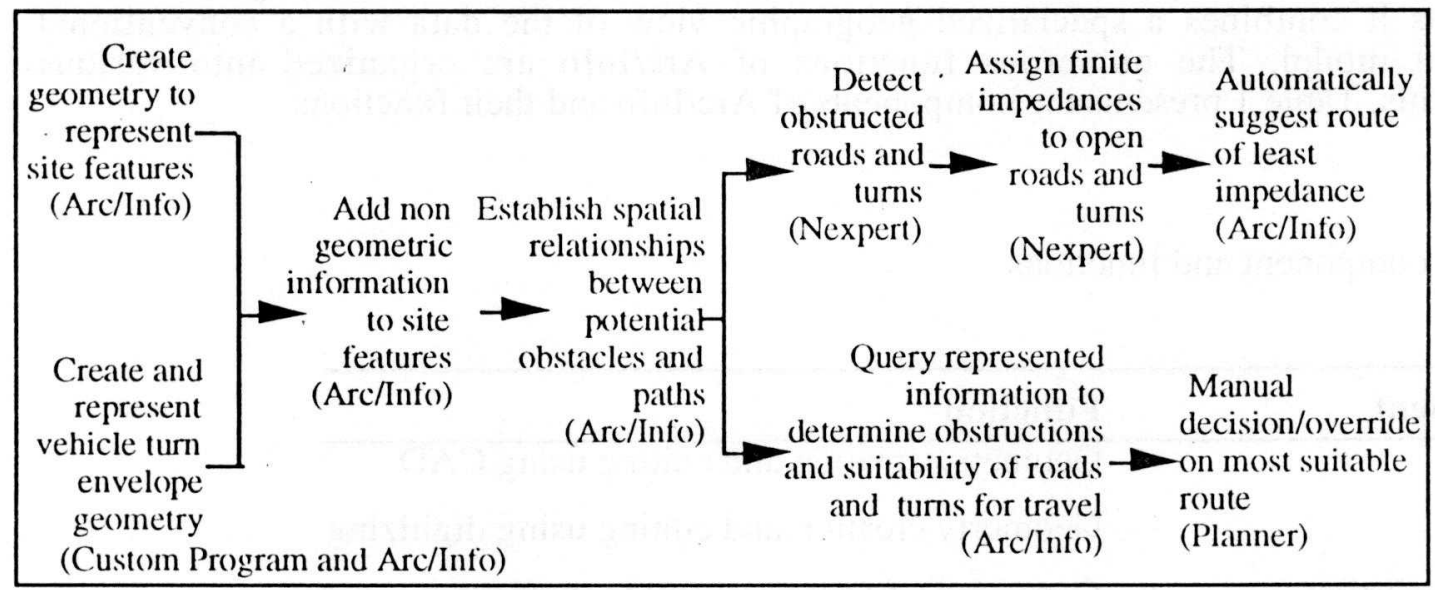

Figure 4. Overall system flow chart

The first step is to develop an integrated representation of the information needed for analysis. Information for a given scenario consists of the layout of the site, construction schedule, facility design and vehicle characteristics. The site layout requires a graphic representation of all the roads, overhead elements, underground elements, permanent facilities and temporary facilities. In addition, the non graphic attributes (design, schedule) of each of these features have to be integrated along with the graphic attributes. The creation, representation and integration of this information is carried out using the basic functions of Arc/Info.

Vehicle characteristics required for analysis consists of its dimensions, weight, kinematics and load distribution scheme. This information is used to test the accessibility of the vehicle on the roads and turns. To test access at a turn, envelopes representing the space required to make the turn have to be generated. The geometry of the turn envelopes is generated by a custom program and this geometry is then represented in an Arc/Info format.

The second step is to establish spatial relationships between the potential obstacles and access paths. The following four spatial relationships have to be identified to determine the potential obstacles on the roads and turns: (i) roads crossed by overhead elements, (ii) roads crossed by underground elements, (iii) turns blocked by permanent facilities, and (iv) turns blocked by temporary facilities. These relationships are established using functions within Arc/Info's Overlay module. It should be noted that the result of these operations are based only on the geometric relationship between the features.

The next step is implemented using a rulebase in the Nexpert environment. In this step the schedule and design information associated with the site features are used to determine 
the roads and turns which are obstructed when the module is delivered to the site. These roads and turns are assigned an infinite impedance to indicate that the route cannot pass through them. Each of the open roads and turns are then assigned a finite impedance based on its characteristics and the importance assigned to each characteristic. The importance assigned to each characteristic depends on the planners preferences and has to be established prior to the analysis and stored as relative weights in the rule base.

Functions to search the road network to select the route of least impedance can be implemented using Arc/Info's Network component. In addition to selecting the route of least impedance, routines within Network can be used to test user defined routes within the site, generate alternate routes and display the routes.

In addition to the functions described above, provision of a querying capability will allow the system to act in a decision support mode. The querying capabilities of the system are implemented using Arcplot. In the decision support mode the planner can query the represented information, view the results graphically to reach a manual decision if necessary. For example, in response to a query to display the permanent facilities which will obstruct the vehicle at a turn, the system initially selects the set of permanent facilities installed before the specified travel day, and from this set those facilities which are within the vehicle's turn envelopes will be displayed in red.

The final component of the system is its user interface. The interface links both software environments to the user and is structured as a hierarchical menu. This interface is developed using Arc/Info's macro language. Figure 5. shows the overall design of the system. Only an overview of the system and it's basic functions are presented in this paper. A detailed description of the system and test problems used to assess the system are given in [8].

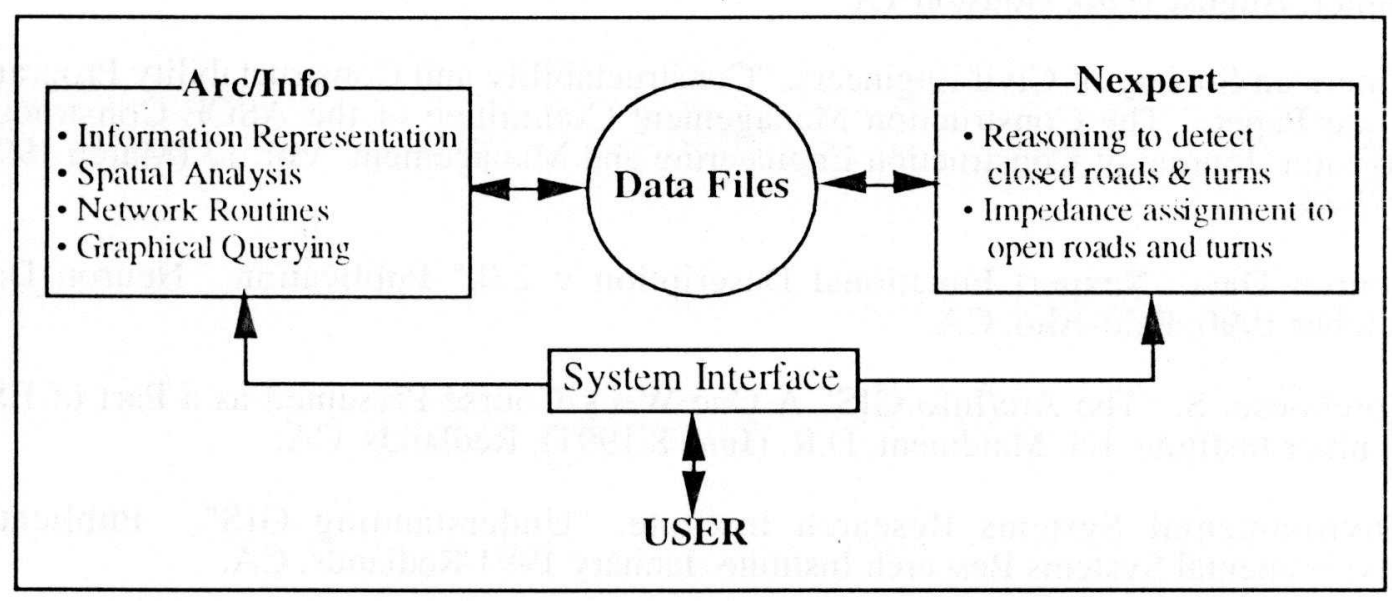

Figure 5. System Design

\section{CONCLUSIONS}

Planning access routes for large vehicles within a construction site is an important consideration in the development of an effective project execution plan. The constructability and the maintainability of a project can be impaired if access routes are not planned properly. But a majority of the planning effort is spent on ensuring no errors are made in carrying out the tedious and repetitive components of the planning process. The 
automation of these components will allow the planner to focus on the creative planning tasks thus enhancing the reliability of the plans and improving planning productivity.

This paper presented an approach to automate the tedious and repetitive tasks required to plan for access on a dynamic construction site. The expert GIS approach described in this work allows the creation of an integrated database which can represent both the graphical and thematic information required for access planning. Using this representation as a base, custom programs, functions within Arc/Info and a rule base were developed to automate the necessary planning tasks.

As constructability analysis generally requires integrated representation of information, spatial analysis and reasoning, this technology can be applied to automate a variety of planning tasks. Further, the tools used for this work provide a suitable environment for the development of custom applications with a minimum investment in time and programming effort.

Future work in this area will focus on expanding the system to: (i) Model uncertainty and identify areas of risk, (ii) Develop an example based pre-processor for quantifying planners preferences, and (iii) Represent strategies and knowledge required to modify a scenario to facilitate access.

\section{REFERENCES}

[1] Construction Industry Institute. "Constructability concepts file". Publication 3-1, Construction Industry Institute, December 1987, The University of Texas at Austin, Austin TX.

[2] Gosch, Donnie Sr. Rigging Consultant, Brown \& Root Braun, Personal Interview By Author, August 1990, Houston TX

[3] American Society of Civil Engineers. "Constructability and Constructability Programs: White Paper." The Construction Management Committee of the ASCE Construction Division. Journal of Construction Engineering and Management Vol. 17 (March 1991): p.67-89.

[4] Neuron Data "Nexpert Functional Description v 2.0." Publication, Neuron Data, October 199(), Palo-Alto, CA.

[5] Morehouse, S. "The Arc/Info GIS" A One Week Course Presented as a Part of ESRI Summer Institute Ed. Maidment, D.R. (June 8 1991): Redlands, CA.

[6] Environmental Systems Research Institute. "Understanding GIS". Publication Environmental Systems Research Institute, January 1991 Redlands, CA.

[7] Maidment, D. R. and Djokic, D.[1991] "Creating an Expert Geographic Information System: The Arc-Nexpert Interface." A One Week Course Presented as a Part of ESRI Summer Institute Ed. Maidment, D.R. (June 8 1991): Redlands, CA.

[8] Varghese, K. "Automated Route Planning For Large Vehicles on Industrial Construction Sites". Dissertation, University of Texas at Austin, Department of Civil Engineering., August 1992. 